\title{
Isoenzyme der Hypoxanthin-Guanin-Phosphoribosyltransferase in menschlichen Erythrocyten
}

\author{
Von M. M. Müller, H. Dobrovits und H. Stemberger \\ Mediziniscb-Cbemisches Institut (Vorstand: Prof. Dr. F. Seelich) und \\ Hygiene-Institut (Vorstand: Prof. Dr. H. Flamm) der Universität Wien
}

(Eingegangen am 7. Juni /8. August 1972)

Es konnte gezeigt werden, daß Hypoxanthin-Guanin-Phosphoribosyltransferase des menschlichen Erythrocyten 4 Isoenzyme besitzt. Diese Isoenzyme, die sich durch ihre elektrophoretischen Eigenschaften auf Polyacrylamidgel unterscheiden, zeigen immunologisch im Doppeldiffusionstest komplette Identität. Als Ursache für die Existenz dieser Isocnzyme wird eine posttranskriptionale Konformationsänderung angenommen.

\section{Isoenzy'mes of buman erytbrocyte bypoxantbine-guanine phosphoribosyltransferase}

The existence of four isoenzymes of hypoxanthine-guanine phosphoribosyltransferase in human erythrocytes could be demonstrated. These isoenzymes, differing in their electrophoretic mobilities on polyacrylamide gel, show complete immunological identity in the double diffusion technique. The existance of four isoenzymes might be due to posttranscriptional alteration of the polypeptide conformation.

Die Purinphosphoribosyltransferasen katalysieren die Umwandlung der Purinbasen zu ihren Mononucleotiden unter Beteiligung von 5-Phosphoribosyl-1-pyrophosphat (1). Dieser sogenannte „Salvage Pathway“ spielt in der Regulation der de novo Purinsynthese eine große Rolle, da die gebildeten Nucleotide den ersten und geschwindigkeitsbestimmenden Schritt hemmen (2). In Bakterien konnten vier verschiedene Purinphosphoribosyltransferasen für die Basen Adenin, Guanin, Hypoxanthin und Xanthin nachgewiesen werden (3). Beim Menschen gibt es hingegen nur zwei Enzyme: die Adenin-Phosphoribosyltransferase (AMP: Pyrophosphatphosphoribosyltransferase; EC 2.4.2.7), die die Umwandlung von Adenin zu Adenosin-5-phosphat (AMP) katalysiert und die Hypoxanthin-Guanin-Phosphoribosyltransferase (IMP: Pyrophosphatphosphoribosyltransferase; EC 2.4.2.8), die Inosin-5-phosphat (IMP), Guanosin-5-phosphat (GMP) und in viel geringerem Maße auch Xanthosin-5-phosphat (XMP) aus den entsprechenden Basen bildet (4).

Bei Kindern mit LESCH-NYHAN-Syndrom wurde ein komplettes Fehlen einer Hypoxanthin-Guanin-Phosphoribosyltransferase-Aktivität nachgewiesen (5), bei manchen Patienten mit Gicht findet sich verminderte Aktivität des Enzyms (6). Dadurch werden die Purinbasen vermindert oder gar nicht $z u$ den Nucleotiden aufgebaut, wodurch die Feedback-Hemmung der Glutamin-Phosphoribosylpyrophosphat-Amidotransferase (EC 2.4.2.14), die den ersten Schritt der Purinsynthese katalysiert, ausbleibt, was die gesteigerte Purinsynthese und somit die hohe Hyperuricaemie bei diesen Patienten erklärt (7).

Kürzlich konnte gezeigt werden, daß ErythrocytenHypoxanthin-Guanin-Phosphoribosyltransferase keinen einheitlichen Eiweißkörper darstellt, sondern aus 3 oder 4 Isoenzymen besteht $(8,9,10)$. Das Ziel der vorliegen- den Studie ist es, bei einer aus Erythrocyten gereinigten Enzympräparation mittels Polyacrylamidgel-Elektrophorese die Isoenzyme darzustellen und mit einem spezifischen Enzym-Antiserum immunologisch zu charakterisieren.

\section{Material und Methoden}

Enzymreinigung

$500 \mathrm{ml}$ Blut eines männlichen Blutspenders mit normaler Hypoxanthin-Guanin-Phosphoribosylțransferase-Aktivität der Erythrocyten dienten als Ausgangsmaterial. Die einzelnen Reinigungsschritte wurden bei $4^{\circ} \mathrm{C}$ vorgenommen. Die Erythrocyten wurden vom Vollblut durch $30 \mathrm{~min}$ Zentrifugation bei $13000 \mathrm{~g}$ abgetrennt und anschließend 3 bis $4 \mathrm{mal}$ mit gleichem Volumen physiol. $\mathrm{NaCl}$-Lösung gewaschen. Durch Tieffrieren bei $-30^{\circ} \mathrm{C}$ und Auftauen bei Raumtemperatur wurden die Erythrocyten hämolysiert.

Die weitere Enzymreinigung entspricht in etwas modifizierter Form der von KRENITSKY (11) angegebenen Methode:

1. Das Hämolysat wurde auf ein $\mathrm{pH}$ von 6,0 eingestellt. Es wurde mit gleichem Volumen einer Suspension von CM-Sephadex C-50 der Firma Pharmacia Uppsala (50 g CM-Sephadex C-50 in 1 Liter Tris- $(10 \mathrm{mmol} / \mathrm{l})$ Magnesiumsulfat- $(2,5 \mathrm{mmol} / \mathrm{l})$ Acetat$(16 \mathrm{mmol} / \mathrm{l})$ Puffer, $\mathrm{pH} 6,0)$ versetzt und $20 \mathrm{~min}$ unter Eiskühlung gerührt. Anschließend wurde die Suspension $10 \mathrm{~min}$ bei 13000 \& zentrifugiert. Der Uberstand wurde noch 3 bis $4 \mathrm{mal}$ mit CMSephadex C-50 Suspension in gleicher Art behandelt, bis er kein Hämoglobin mehr enthielt.

2. Zur Entfernung von Adenin-Phosphoribosyltransferase und hitzelabilen Proteinen wurde der Uberstand im Wasserbad auf $73^{\circ} \mathrm{C}$ erhitzt, $5 \mathrm{~min}$ bei $73^{\circ} \mathrm{C}$ gehalten, gekühlt und das denaturierte Eiweiß abfiltriert. Das Filtrat wurde gefriergetrocknet und in $4 \mathrm{ml} 0,02 \mathrm{~mol} / \mathrm{l}$ Tris-HCl-Puficr $(\mathrm{pH} 7,7)$, der $0,2 \mathrm{mmol} / \mathrm{l}$ Magnesiumsulfat enthält, aufgenommen.

3. Die Probe $(4 \mathrm{ml})$ wurde auf einc Sephadex G-100 Säulc $(33 \times 2 \mathrm{~cm})$ aufgetragen und mit Tris-HCl-Puffer ( $\mathrm{pH}$ 7,7; $0,2 \mathrm{mmol} / 1 \mathrm{MgSO}_{4}$ ) eluicrt. Die Elutionsgeschwindigkeit betrug $15 \mathrm{ml} / \mathrm{h}$. Fraktionen zu $3 \mathrm{ml}$ wurden gesammelt. Fraktionen mit Hypoxanthin-Guanin-Phosphoribosyltransferasc-Aktivität wurden gepoolt und mittcls Centriflo Membranfilter 224-CF-50 (Amicon Corp., Jexington, Mass., USA) konzentriert. 
Hämoglobin wurde nach der Methode von vaN KAMPEN (12) mit Bochringer-Reagenzien, Protein wurde nach der Biuret-Methode bestimmt (13).

\section{Enzymtest}

Zur Bestimmung der Enzymaktivitäten verwendeten wir eine von KELLEY (14) angegebene radiochemische Methode in etwas modifizierter Form (15): Die Endkonzentration der Reagenzien bzw. Substrate betrug: $55 \mathrm{mmol} / 1$ Tris-HCl-Puffer (pH 7,4), $5 \mathrm{mmol} / \mathrm{l} \mathrm{MgCl} 2,0,8 \mathrm{mmol} / 1$ Phosphoribosylpyrophosphat und $0,6 \mathrm{mmol} / 1{ }^{14} \mathrm{C}$-Hypoxanthin $(15,0 \mathrm{mCi} / \mathrm{mmol}$; Radiochemical Center Amersham, England). Die Reaktion wurde mit 0,3-0,6 mg Enzymprotein durchgeführt und nach 20 min Inkubation bei $37^{\circ} \mathrm{C}$ mit $0,6 \mathrm{~mol} / 1 \mathrm{HClO}_{4}$ gestoppt. Das gebildete ${ }^{14} \mathrm{C}$-IMP wurde papierelektrophoretisch abgetrennt und die Radioaktivität im Liquidscintillationcounter gemessen.

\section{Disk-Elektrophorese}

Als Kontrolle der Reinigung und zur Lokalisierung der Enzymaktivität verwendeten wir Säulen von 7,5 proz. Polyacrylamidgel $(4 \times 50 \mathrm{~mm}$; Firma Serva, Heidelberg, BRD) in der von Davrs angegebenen Form (16). Enzymlösung entsprechend 0,6 mg Protein wurde in Saccharoselösung $(100 \mathrm{~g} / \mathrm{l})$ über das Sammelgel geschichtet. Die Trennung erfolgte bei $4^{\circ} \mathrm{C}$ innerhalb von $3 \mathrm{~h}$ bei einer Spannung von 150 Volt (Beckman Spinco Duostat). Als Vergleichssubstanz wurde Bromphenolblau verwendet. Die Gele wurden $20 \mathrm{~min}$ mit gesättigter Amidoschwarz-Lösung in 7proz. Essigsäure gefärbt und mittels 7 proz. Essigsäure entfärbt. Die so dargestellten Eiweißbanden wurden densitometrisch mit einem Vitatron-Densitometer ausgewertet.

Zur Lokalisierung der Enzymaktivitäten und zur Bestimmung der Isoenzyme wurden nicht angefärbte Gele mit Puffer und Substraten $30 \mathrm{~min}$ bei $37^{\circ} \mathrm{C}$ inkubiert. Nach abgelaufener Reaktion in den Gelen wurde jedes Gel in $1 \mathrm{~mm}$ dicke Scheibchen geschnitten, jedes Scheibchen auf einen nassen Elektrophoresestreifen aufgelegt und das Reaktionsprodukt ${ }^{14} \mathrm{C}$-IMP aus dem Gel abgetrennt. Zur immunologischen Charakterisierung der Isoenzyme wurde wieder ein nicht gefärbtes $\mathrm{Gel}$ in $1 \mathrm{~mm}$ dicke Scheibchen geschnitten, jedes Scheibchen in $30 \mu \mathrm{l}$ physiol. $\mathrm{NaCl}$-Lösung homogenisiert, das Homogenat in die Näpfe der OuChterLony-Platten eingegossen und mit Enzym-Antiserum auf seine antigenen Eigenschaften untersucht.

\section{Herstellung des Enzym-Antiserums}

Zur Gewinnung von Enzym-Antisera immunisierten wir 5 männliche Kaninchen mit einem Körpergewicht von etwa $3 \mathrm{~kg}$ mit Enzymlösung.

Zur Erstimmunisierung erhielten die Tiere das Enzym in komplettem FreUnD'schen Adjuvans in die Pfote injiziert, wobei jedem Tier $1 \mathrm{ml}$ Enzym (10 mg Protein/ml), $1 \mathrm{ml}$ physiol. $\mathrm{NaCl}$ Lösung und $2 \mathrm{ml}$ komplettes FreUND'sches Adjuvans verteilt auf alle vier Pfoten injiziert wurde.

Die zweite Antigengabe erfolgte 1 Woche später subcutan an zwei Stellen unter die Rückenhaut. Jedem Tier wurde wieder Antigen entsprechend einer Eiweißmenge von $10 \mathrm{mg}$ in $4 \mathrm{ml}$ Gesamtvolumen, dieses $\mathrm{Mal}$ jedoch mit inkomplettem FREUND'schem Adjuvans, injiziert.

4 Wochen nach Immunisierungsbeginn wurden jedem Tier nochmals $10 \mathrm{mg}$ Antigen in physiol. $\mathrm{NaCl}$-Lösung, jedoch ohne Zusatz von Adjuvantien intravenös verabreicht.

10 Tage darauf erfolgte die Blutabnahme mittels Herzpunktion. Die Sera wurden in kleinen Portionen ohne konservierende $\mathrm{Zu}$ sätze bei $-20^{\circ} \mathrm{C}$ gelagert.

\section{Agargel-Doppeldiffusionstest}

Die Tests erfolgten auf Immuno-Plate (pattern C) der Firma Hyland (Div. Travenol Lab. Inc., Costa Mesa, Calif., USA) (17).

Enzym-Hemmtest mit dem Enzym-Antiserum

Die Hemmung der Hypoxanthin-Guanin-Phosphoribosyltransferase exfolgte durch Zugabe steigender Mengen $(20-100 \mu \mathrm{l})$ Antiserums (3,6 mg Protein/ml) zum Enzymtest. Als Kontroll- wert diente ein auf den Eiweißgehalt des Antiserums eingestelltes normales Kaninchenserum, von dem ebenfalls 20-100 $\mu \mathrm{l}$ dem Test zugesetzt wurden. Die Reaktion wurde wieder $20 \mathrm{~min}$ bei $37^{\circ} \mathrm{C}$ durchgeführt. Die Substratkonzentrationen und die weiteren Schritte der Enzymaktivitätsbestimmung entsprachen den bereits oben beschriebenen Methoden.

\section{Ergebnisse}

\section{Enzymreinigung}

Abbildung 1 zeigt, daß Hypoxanthin-Guanin-Phosphoribosyltransferase nicht als einheitlicher Peak (Enzym-Fraktion) aus der Sephadex G-100 Säule eluiert wird. Der zweite Eiweißgipfel (Begleitproteine) der Elutionskurve entspricht Proteinen ohne nennenswerte Enzymaktivität. Die Enzymaktivität der hämolysierten Erythrocyten betrug $0,083 \mu \mathrm{mol} / 20 \mathrm{~min} \cdot \mathrm{mg}$ Protein, die der eingeengten Fraktionen des Enzympeaks $5,88 \mu \mathrm{mol} / 20 \mathrm{~min} \cdot \mathrm{mg}$ Protein. Das entspricht einer etwa 70-fachen Enzymanreicherung gegenüber dem Hämolysat. Weder im ersten noch im zweiten Eiweißpeak waren nach Einengung im Agargel-Doppeldiffusionstest mit Human-Antiserum Serumproteine nachzuweisen.

\section{Immunologische Untersuchungen}

Die Immunisierung der Kaninchen mit den Enzymlösungen führte zum Auftreten von präzipitierenden Antikörpern. Es zeigt sich im Doppeldiffusionstest nach Ouchterlony (Abb. 2), daß die Enzym-Fraktion mit Enzym-Antiserum 302 zwei Präzipitationslinien erkennen läßt. Die Begleitproteine hingegen geben in dieser Versuchsanordnung nur eine Präzipitationsbande, die mit der dem Antigentrog näher gelegenen Iinie der Enzym-Fraktion fusioniert.

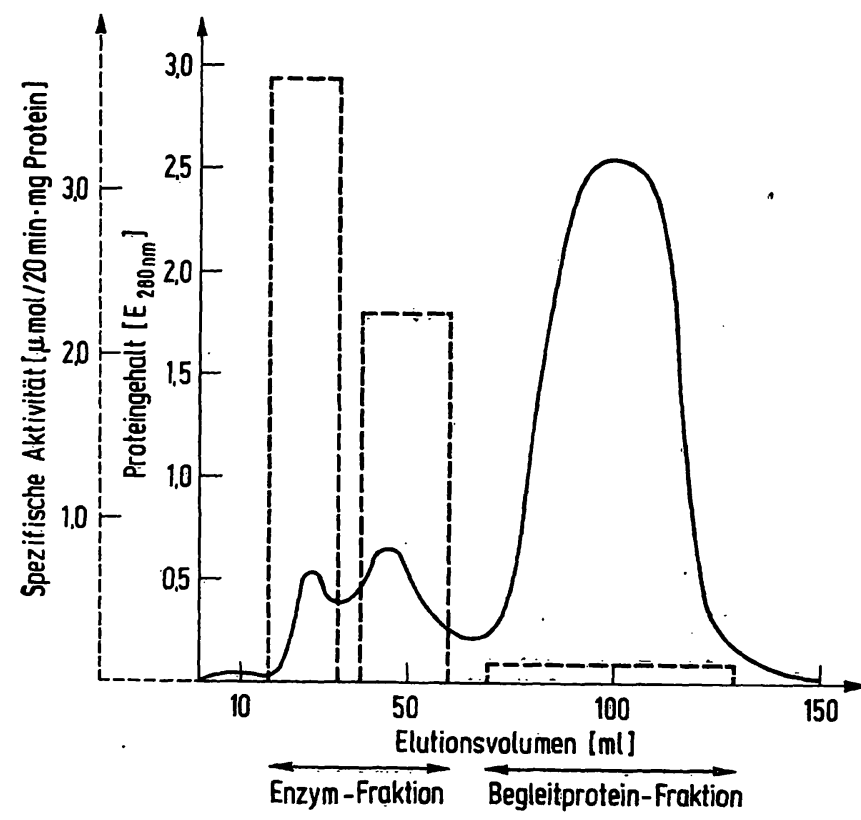

Abb. 1

Sephadex G-100-Chromatographie der Hypoxanthin-Guanin-Phosphoribosyltransferase

Elutionskurve der Proteine (Messung der Extinktion bei $280 \mathrm{~nm}$ ); 


\section{Bei der Beschaffung von Derivaten von cyclischem 3':5'-AMP kann Ihnen keine andere Firma so gut helfen wie Sigma}

Das erste käuflich erhältliche cyclische $3^{\prime}: 5^{\prime}$-AMP wurde vor über 10 Jahren von Sigma angeboten. Wir stellten es durch alkalische Hydrolyse von ATP mit etwa $2 \%$ Ausbeute mühsam her. Es kostete damals $\$ 500 / \mathrm{g}$. Heute bieten wir unsere Nr. A 9501 mit einer Reinheit von etwa $99 \%$ in 25 g-Packun-

A 9501

Adenosin-3':5'-monophosphorsăure, cyelisch, freie Säure

gen für weniger als $\$ 20 / g$ an.

\begin{tabular}{rrr}
$25 \mathrm{mg}$ & $\$$ & 2,00 \\
$100 \mathrm{mg}$ & $\$$ & 5,40 \\
$500 \mathrm{mg}$ & $\$$ & 18,00 \\
$1 \mathrm{~g}$ & $\$$ & 29,00 \\
$5 \mathrm{~g}$ & $\$ 136,00$ \\
$25 \mathrm{~g}$ & $\$ 485,00$ \\
\hline $25 \mathrm{mg}$ & $\$$ & 4,50 \\
$50 \mathrm{mg}$ & $\$$ & 7,50 \\
$100 \mathrm{mg}$ & $\$$ & 12,60 \\
$250 \mathrm{mg}$ & $\$$ & 25,20 \\
$500 \mathrm{mg}$ & $\$ 42,05$ \\
$1 \mathrm{~g}$ & $\$ 69,50$ \\
$5 \mathrm{~g}$ & $\$ 325,00$
\end{tabular}

Sigma bietet auch einige verwandte AMP-Derivate an, die wir für sehr gut halten

\begin{tabular}{|c|c|c|}
\hline $\begin{array}{l}\text { B } 7880 \\
\text { 8-Bromadenosin-3': 5'-monophosphorsäure, } \\
\text { cylisch, Natriumsalz }\end{array}$ & $\begin{array}{r}2 \mathrm{mg} \\
5 \mathrm{mg} \\
25 \mathrm{mg} \\
100 \mathrm{mg} \\
250 \mathrm{mg}\end{array}$ & $\begin{array}{rr}\$ 5,50 \\
\$ 10,80 \\
\$ 36,00 \\
\$ 100,00 \\
\$ 200,00\end{array}$ \\
\hline $\begin{array}{l}\text { D } 1256 \\
\text { 2'-Desoxyadenosin-3':5'-monophosphorsäure, } \\
\text { cyclisch, Natriumsalz }\end{array}$ & $\begin{array}{r}5 \mathrm{mg} \\
10 \mathrm{mg} \\
25 \mathrm{mg} \\
100 \mathrm{mg} \\
250 \mathrm{mg}\end{array}$ & $\begin{array}{lr}\$ & 8,40 \\
\$ & 13,70 \\
\$ & 27,50 \\
\$ & 75,00 \\
\$ & 150,00\end{array}$ \\
\hline $\begin{array}{l}\text { D } 0627 \\
N^{0}, \text { O't'-Dibutyryl-adenosin- }^{\prime} \\
\text { 3': }^{\prime} \text { '-monophosphorsäure, } \\
\text { cyclisch, Natriumsalz } \\
\vdots\end{array}$ & $\begin{array}{l}5 \mathrm{mg} \\
25 \mathrm{mg} \\
100 \mathrm{mg} \\
250 \mathrm{mg} \\
1 \mathrm{~g}\end{array}$ & $\begin{array}{rr}\$ & 3,00 \\
\$ & 9,00 \\
\$ & 23,50 \\
\$ & 45,00 \\
\$ & 149,00\end{array}$ \\
\hline $\begin{array}{l}\text { M } 1380 \\
N^{0}-\text { Monobutyryl-adenosin- } \\
3^{\prime}: 5^{\prime} \text {-monophosphorsäure, } \\
\text { cyclisch, Nafriumsalz }\end{array}$ & $\begin{array}{r}2 \mathrm{mg} \\
5 \mathrm{mg} \\
10 \mathrm{mg} \\
25 \mathrm{mg} \\
100 \mathrm{mg}\end{array}$ & $\begin{array}{lr}\$ & 4,60 \\
\$ & 7,80 \\
\$ & 13,00 \\
\$ & 27,50 \\
\$ & 100,00\end{array}$ \\
\hline $\begin{array}{l}\text { M } 1505 \\
\text { O'-Monobutyryl-adenosin- }^{2 \prime} \text { 3':5'-monophosphorsäure, } \\
\text { cyclisch, Natriumsalz }\end{array}$ & $\begin{array}{r}2 \mathrm{mg} \\
5 \mathrm{mg} \\
10 \mathrm{mg} \\
25 \mathrm{mg} \\
100 \mathrm{mg}\end{array}$ & $\begin{array}{lr}\$ & 9,70 \\
\$ & 20,00 \\
\$ & 32,00 \\
\$ & 64,00 \\
\$ 175,00\end{array}$ \\
\hline
\end{tabular}

\begin{tabular}{|c|c|c|}
\hline $\begin{array}{l}\text { M } 3256 \\
O^{2 \prime} \text {-Monobutyryl-8-bromo-adenosin- } \\
\text { 3':5'-monophosphorsäure, } \\
\text { cyclisch, Natriumsalz }\end{array}$ & $\begin{array}{r}2 \mathrm{mg} \\
5 \mathrm{mg} \\
25 \mathrm{mg} \\
100 \mathrm{mg}\end{array}$ & $\begin{array}{l}\$ 15,00 \\
\$ 27,50 \\
\$ 90,00 \\
\$ 250,00\end{array}$ \\
\hline $\begin{array}{l}\text { M } 8381 \\
\text { No-Monobulyryl-2-desoxyadenosin- } \\
\text { 3':5'-monophosphorsdiure, } \\
\text { cyclisch, Natriumsalz }\end{array}$ & $\begin{array}{r}2 \mathrm{mg} \\
5 \mathrm{mg} \\
25 \mathrm{mg} \\
100 \mathrm{mg}\end{array}$ & $\begin{array}{l}\$ 15,00 \\
\$ 27,50 \\
\$ 90,00 \\
\$ 250,00\end{array}$ \\
\hline $\begin{array}{l}\text { M } 3006 \\
O^{3 \prime} \text {-Monobutyryl-guanosin- } \\
\text { 3':5'-monophosphorsäure, } \\
\text { cyclisch, Natirumsalz }\end{array}$ & $\begin{array}{r}2 \mathrm{mg} \\
5 \mathrm{mg} \\
10 \mathrm{mg} \\
25 \mathrm{mg} \\
100 \mathrm{mg}\end{array}$ & $\begin{array}{r}9,75 \\
\$ 20,00 \\
\$ 32,00 \\
\$ 64,00 \\
\$ 175,00\end{array}$ \\
\hline 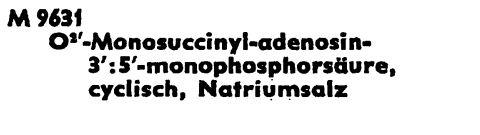 & $\begin{array}{r}2 \mathrm{mg} \\
5 \mathrm{mg} \\
10 \mathrm{mg} \\
25 \mathrm{mg} \\
100 \mathrm{mg}\end{array}$ & $\begin{array}{r}30,00 \\
\$ 54,00 \\
\$ 90,00 \\
\$ 180,00 \\
\$ 500,00\end{array}$ \\
\hline $\begin{array}{l}\text { M } 9131 \\
\text { O'_-Monosuccinyl-adenosin- }^{\prime \prime} \text { 3':5'-monophosphorsäure, } \\
\text { cyclisch, freie Säure }\end{array}$ & $\begin{array}{r}2 \mathrm{mg} \\
5 \mathrm{mg} \\
10 \mathrm{mg} \\
25 \mathrm{mg} \\
100 \mathrm{mg}\end{array}$ & $\begin{array}{r}30,00 \\
\$ 54,00 \\
\$ 90,00 \\
\$ 180,00 \\
\$ 500,00\end{array}$ \\
\hline
\end{tabular}

Zusätzlich sind neben dieser Haupłkategorie folgende Verbindungen erhältlich:

Wir hoffen, außerdem demnächst anbieten zu können:

8-Aminoadenosin-3':5'-monophosphorsäure, cyclisch 8-Methoxyadenosin-3': $5^{\prime}$-monophosphorsäure, cyclisch 8-Methylthioadenosin- $3^{\prime}: 5^{\prime}$-monophosphorsäure, cyclisch 8-Methylthioadenosin-3' $15^{\prime}$-monophosphorsäure, cyclisch
8-Äthylthioadenosin-3': $5^{\prime}$-monophosphorsäure, cyclisch 8-Athylthioadenosin-3': $5^{\prime}$-monophosphorsäure, cyclisch
8-Methylaminoadenosin $-3^{\prime}: 5^{\prime}$-monophosphorsäure, cyclisch

Tyrosyl-methylester von $0^{2}$-Monosuccenyl-adenosin- $3^{\prime}: 5^{\prime}$-monophophorsäure, cyclisch

$$
\begin{aligned}
& \text { 8-Hydroxyadenosin- } 3^{\prime}: 5^{\prime}-\text { monophosphorsäure, cyclisch } \\
& N^{2}, O^{2} \text {-Dibutyrylguanosin- } 3^{\prime}: 55^{\prime} \text {-monophosphorsäure, cyclisch }
\end{aligned}
$$
$N^{6}, O^{2}$-Disuccinyl-adenosin-3':5'-monophosphorsä
Z-Azidodenosin- $3^{\prime}: 5^{\prime}$-monophosphorsäure, cyclisch

Da einige Forscher Mikromengen von cyclischem $3^{\prime}: 5^{\prime}$-AMP bestimmen möchten, haben wir kleine Mengen von Proteinkinase (von cyclischem $3^{\prime}$ :5'-AMP abhängig) und Proteinkinase-Inhibitor nach A. Gilman, Proc. Nat. Acad. Sci. 61, 305 (1970) bereitgestellt.

Da die „,kleine“ Sigma bisher nicht entsprechend ausgerüstet ist, mußten wir die Zusammenarbeit mit einem fremden Laboratorium zur Bestimmung dieser Produkte in Anspruch nehmen. Uns wurde berichtet, daß $1 \mu \mathrm{g}$ Proteinkinase (von cyclischem $3^{\prime}: 5^{\prime}$-AMP abhängig, Charge $22 \mathrm{C}-8540$ ) bei $0^{\circ} \mathrm{C}$ und pH 40,05 pmol cyclisches 3':5'-AMP bindet. Durch Zugabe des Inhibitors wird die Bindungskapazitäł verdoppelt.

P 5511

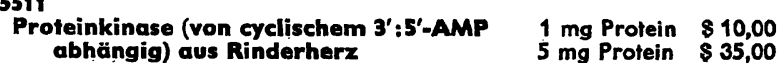

Lyophilisiertes Pulver, enthält etwa $80 \%$ Protein, 10\% EDTA und $10 \%$ Kaliumphosphat, pH 7. Enthält v. a. folgende Verunreinigungen:

$<0,01 \mathrm{U} / \mathrm{mg}$ ATPase und etwa 0,5 U/mg Phosphodiesterase und cyclisches $3^{\prime}: 5^{\prime}$-Nucleotid, das unter Bedingungen des Binding Assay inaktiv ist

\begin{tabular}{ll}
\hline 5636 & $10 \mathrm{mg}$ Protein $\$ \mathbf{7 , 5 0}$ \\
Proteinkinase-Inhibitor aus Rinderherz & $50 \mathrm{mg}$ Protein $\$ 25,00$
\end{tabular}

Lyophilisiertes Pulver, etwa $98 \%$ Protein und < 2\% Puffersalze Alle Preise einschließlich normaler Versandgebühr zu-lhnen überall hin in die Welt.

Es ist ein Vergnügen, Geschäfte mit Sigma zu machen

Bestellen Sie direkt - R-Gespräch von überali her in der Welt Tagsüber von Haus zu Haus, 314-771-5750. Nachts von Person zu Person, Dan Broida, 314-092-6418 TWX (Fernschreiber) Tag und Nacht: 910-761-0593 Telegramme: SIGMACHEMI, St. Louis, Missouri

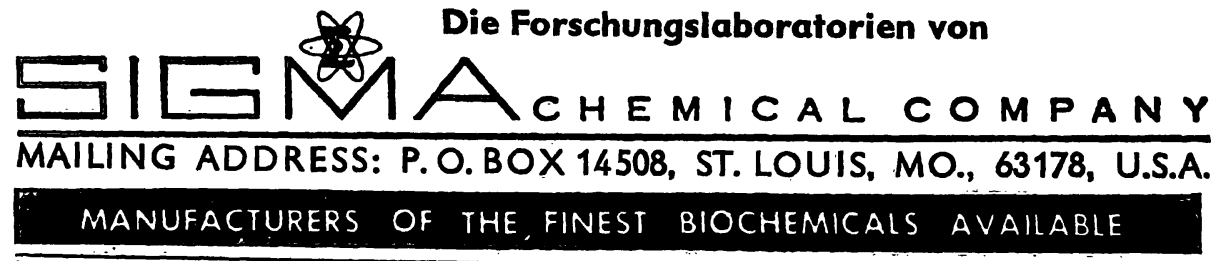




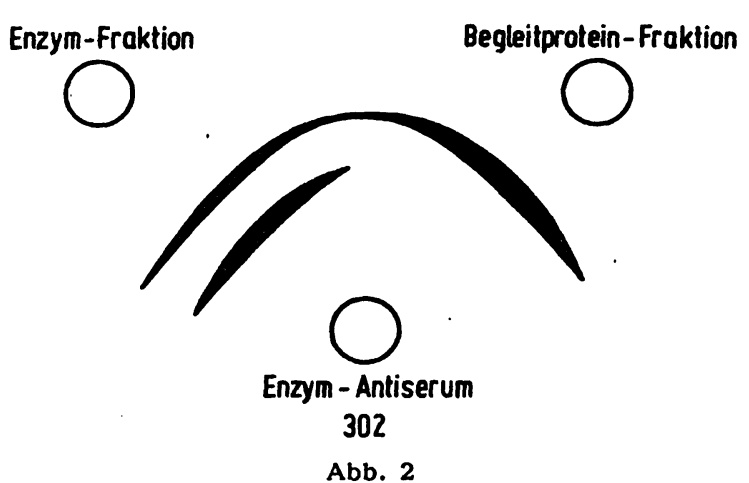

Agargel-Doppeldiffusionstest der eingeengten Fraktionen mit Enzym-
Antiserum

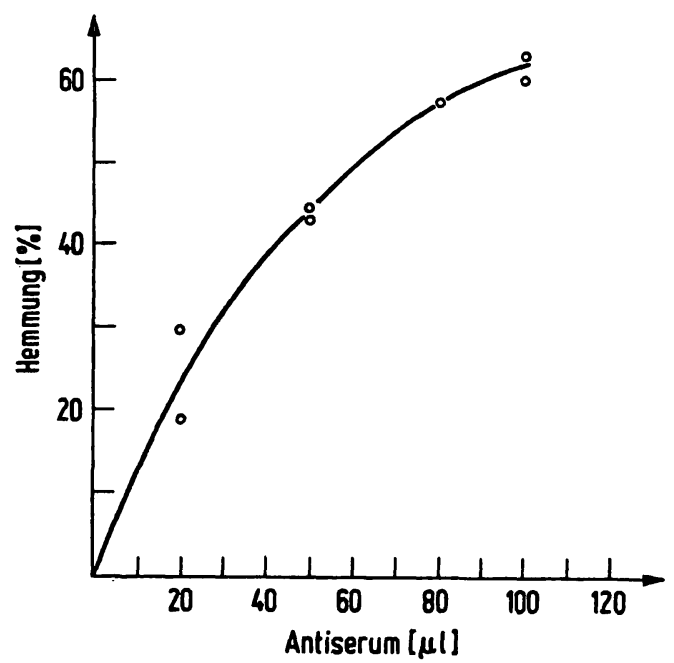

Abb. 3

Enzymhemmung mit Enzym-Antiserum

\section{Enzym-Hemmtest}

Um die biologische Wirksamkeit der gewonnenen Antikörper zu untersuchen, führten wir mit dem Enzym-Antiserum 302 einen Hemmtest durch. Bei konstanter Enzymkonzentration konnte mit Antiserum eine dosisabhängige Hemmung der Enzymaktivität beobachtet werden (Abb. 3). Die entsprechen- den Kontrollversuche mit normalem Kaninchenserum zeigten keine Enzymhemmung.

\section{Untersuchungen der Isoenzyme}

Mittels der Polyacrylamidgel-Disk-Elektrophorese führten wir eine weitere Auftrennung der in der EnzymFraktion enthaltenen Proteine durch. Bei Eiweißfärbung zeigten sich 3 scharf getrennte Banden (Abb. 4). Um festzustellen, ob die gefundenen Eiweißbanden Enzymaktivität enthalten, inkubierten wir nicht gefärbte Gele mit Substrat: 4 verschiedene Aktivitätsmaxima (Isoenzyme 1-4) stellten sich auf diese Weise dar (Abb. 4). Die Aktivitätsmaxima lagen.im Bercich der Eiweißbanden. Dem Isoenzym 1 und 2 entspricht die am weitesten anodisch wandernde Eiweißbande. Das Eiweißäquivalent zu Isoenzym 3 deckt sich genau mit dessen Aktivität. Es fiel allerdings auf, daß die dem Isoenzym 4 entsprechende Eiweißbande sich kathodisch über deren Aktivitätsbcreich hinaus erstreckt.

Die im Agargel-Doppeldiffusionstest der einzelnen Gelabschnitte mit Enzym-Antiserum 302 darstellbaren Präzipitationslinien wiesen auf das Vorhandensein antigenen Materials hin, dessen Verteilungsmuster sowohl den einzelnen Isoenzymaktivitäten als auch den Eiweißbanden entsprach (Abb. 4). In den Gelscheibchen entsprechend Wanderungsquotienten von 0,285 bis 0,432 traten 2 Präzipitationslinien auf, in allen übrigen antigenhältigen Gelabschnitten hingegen nur eine.

Zur Feststellung der Identität der Präzipitationsbanden und damit zur immunologischen Charakterisierung der Isoenzyme untersuchten wir in weiteren Diffusionstests die den Isoenzymmaxima entsprechenden Zonen der Polyacrylamidgele und verglichen sie mit der EnzymFraktion nach der in Abbildung 5 wiedergegebenen Anordnung. Alle Isoenzymscheibchen zeigten bei diesem Versuch eine Präzipitationslinie, die mit der der Auftragungsstelle des Enzym-Antiserums näher gelegenen Linie der Enzym-Fraktion identisch war. Die im Isoenzympeak 1 aufgetretene zweite, dem Antigen-

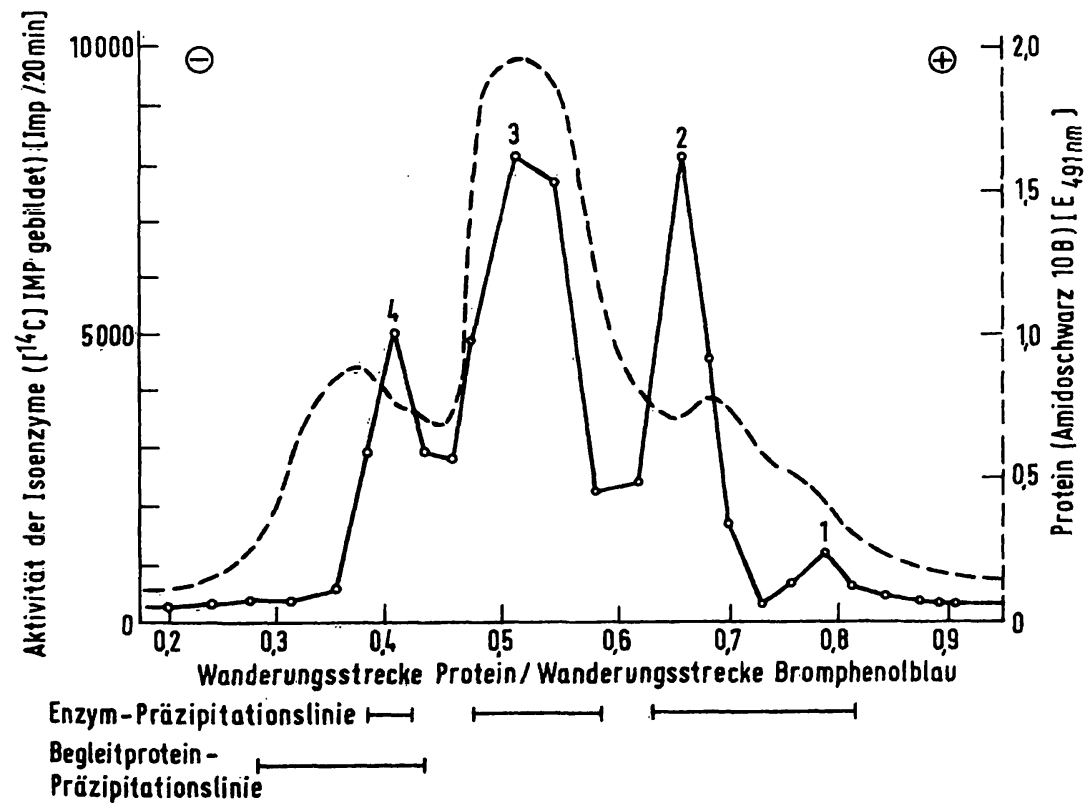

Abb. 4

Isoenzymmuster der Hypoxanthin-Guanin-Phosphori-

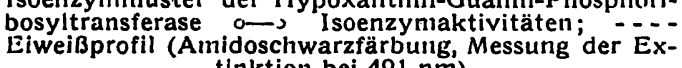
tinktion bei $491 \mathrm{~nm}$ ) 


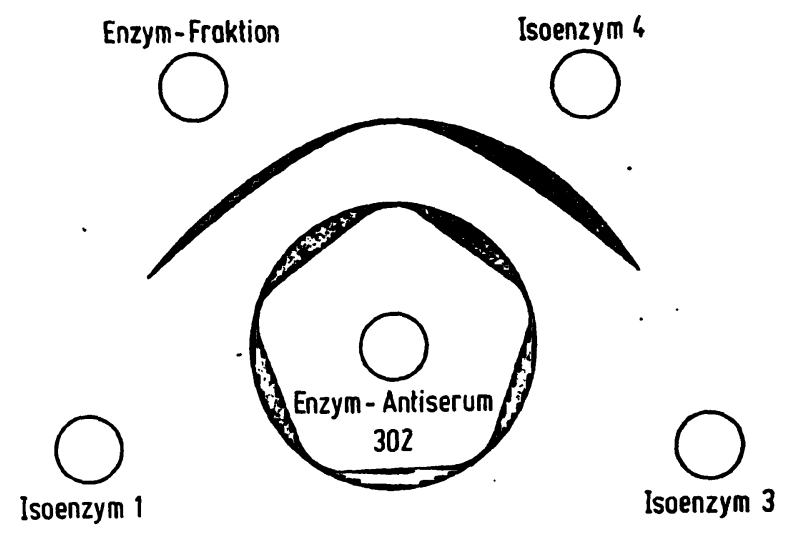

Abb. 5
Agargel-Doppeldiffusionstest der Hypoxanthin-Guanin-Phosphoribo-
syltransferase-Isoenzyme mit Enzym-Antiserum

auftragungsort näher gelegene Präzipitationslinie ergab mit den Begleitproteinen der Enzym-Fraktion eine komplette Identitätsreaktion.

\section{Diskussion}

Von den nach Sephadex-Chromatographie erhaitenen beiden Fraktionen besitzt praktisch nur die EnzymFraktion, mit der immunisiert wurde, eine Hypoxanthin-Guanin-Phosphoribosyltransferase-Aktivität (Abb. 1). Die Agargel-Doppeldiffusionstests ergaben das Vorliegen eines gemeinsamen Proteins in beiden Fraktionen, das mit Hypoxanthin-Guanin-Phosphoribosyltransferase nicht identisch ist (Begleitproteine). Die zweite, nur in der Enzym-Fraktion auftretende Präzipitationslinie ist mit größter Wahrscheinlichkeit das Reaktionsprodukt von Hypoxanthin-Guanin-Phosphoribosyltransferase mit dem Enzym-Antiserum. Die über 60proz.
Hemmung der Enzymaktivität durch das Antiserum unterstreicht dessen spezifische Wirkung.

Die Enzym-Fraktion, die anhand der Diffusionsteste das Vorhandensein von zwei Komponenten vermuten ließ, zeigte hingegen in der Auftrennung mittels Polyacrylamidgel-Elektrophorese 3 Eiweißbanden und 4 Enzymaktivitätsmaxima, entsprechend den 4 Isoenzymen. In allen Gelabschnitten mit Enzymaktivität ließ sich in der Doppeldiffusion die Enzymlinie (Abb. 5) einwandfrei nachweisen. Dic in der Enzym-Fraktion immunologisch nachgewiesenen Verunreinigungen konnten auch bei dieser Versuchsanordnung nachgewiesen werden und korrelierten gut mit den Eiweißbanden.

Unsere Ergebnisse zeigen, daß Erythrocyten-Hypoxanthin-Guanin-Phosphoribosyltransferase 4 Isoenzyme besitzt, die sich durch ihre elektrophoretischen Eigenschaften deutlich unterscheiden, die aber immunologisch im Doppeldiffusionstest komplette Identität aufweisen.

Das immunologische Verhalten der Isoenzyme spricht für eine posttranskriptionale Änderung der am gleichen Gen synthetisierten Polypeptidkette des Enzymproteins (18). Ob diese Änderung durch Aufspaltung des oligomeren Enzyms zu lsoenzymen mit unterschiedlichen Polypeptidketten, durch Bildung von Konformationsisomeren oder durch Reaktion niedermolekularer Modulatoren verursacht wird, kann anhand der vorliegenden Befunde noch nicht gesagt werden. Zur endgültigen Klärung der Frage nach der Entstehung der Isoenzyme der Hypoxanthin-Guanin-Phosphoribosyltransferase ist eine weitere Reinigung mit anschließenden enzymkinetischen Untersuchungen notwendig.

Von großem klinischen Interesse ist die Frage, ob Patienten mit Hyperuricaemie und verminderter Enzymaktivität $(6,15)$ ein verändertes Isoenzymmuster haben, und ob bei Kindern mit LESCH-NYHAN-Syndrom das Enzym tatsächlich fehlt oder ob ein mutiertes inaktives Enzymprotein vorliegt.

\section{Literatur}

1. Korn, E. O., Remy, C. N., Wastleyko, H. C. \& Buchanan, J. M. (1955), J. Biol. Chem. 217, 875-883. - 2. Nierlich, O. P. \& Magasanik, B. (1965), J. Biol. Chem. 240, 358-365. 3. Kalle, G. P. \& Gots, J. S. (1961), Fed. Proc. Fed. Amer. Soc. Exp. Biol. 20, 358. - 4. Kelley, W. N., Rosenbloom, F. M., Henderson, J. F. \& Seegmiller, J. E. (1967), Biochem. Biophys. Res. Commun. 28, 340-345. - 5. SeEgmiller, J. E., RosenBLOOM, F. M. \& Kelley, W. N. (1967), Science 155, 1682-1683. 6. Kellex, W. N., Greene, M. L., Rosenbloom, F. M., Henderon, J. F. \& SEegmiller, J. E. (1969), Ann. Intern. Med. 70, 155-206. 7. Sorensen, L. B. (1970), J. Clin. Invest. 49, 968-978. - 8. Arnold, W. J. \& Kelley, W. N. (1971), J. Biol. Chem. 246, 7398-7404. - 9. Davies, M. R. \& Dean, B. M. (1971), FEBS
Lett. 18, 283-286. - 10. MülLER, M. M. \& DobRovirtrs, H. (1972), Prep. Biochem. (im Druck). - 11. Krenitskx, T. A., Papaioannou, R. \& Elion, G. B. (1969), J. Biol. Chem. 244, 1263-1270. - 12. van Kampen, E. J. \& ZiJLSTRA, W. G. (1961), Clin. Chim. Acta 6, 538-544. - 13. Sols, A. (1947), Nature (London) 160, 89. - 14. KeLley, W. N., Rosenbloom, F. M., Henderon, J. F. \& Seegmiller, J. E. (1967), Proc. Nat. Acad. Sci., USA 57, 1735-1739. - 15. MülleR, M. M. (1972), Fortschritte der klinischen Chemie, Enzyme und Hormone, S. 233 bis 237, Verlag d. Wien. Med. Akademie. - 16. Davis, B. J. (1964), Ann. N. Y. Acad. Sci. 121, 404-427. - 17. OuchterLony, O. (1949), Acta Pathol. Microbiol. Scand. 26, 507-515. - 18. Markert, C. L. (1968), Ann. N. Y. Acad. Sci. 151, $14-40$.

Dr. med. Mathias M. Müller und Dr. med. Heinz Dobrovits Medizinisch-Chemisches Institut der Universität Wien

Währingerstraße 10

A 1090 Wien

Dr. med. Heinrich Stemberger

Hygiene-Institut der Universität Wien

Kinderspitalgasse 15

A 1090 Wien

Österreich/Austria 